\title{
Nitric Oxide Is Involved in the Response of the Isolated Intact and Epithelium-Denuded Rat Trachea to the $\beta_{2}$ Adrenergic Receptor Agonist Salbutamol
}

\author{
F. Montalvo O. Cantres-Fonseca G.Santos M. Vega I. Torres J.Carmona \\ D. Dexter G. Santacana
}

Departments of Physiology and Medicine, Pulmonary Section, University of Puerto Rico School of Medicine, San Juan, P.R., USA

\section{Key Words}

Nitric oxide $\cdot$ Rat trachea $\cdot$ Salbutamol

\begin{abstract}
Background: The involvement of the airway smooth muscle mediator nitric oxide (NO) in the actions of the $\beta_{2}$ agonist salbutamol (Sal), a well- known bronchodilator, is very poorly understood. Objectives: To determine if endogenous NO release is a major factor in the Sal-induced relaxation of the carbachol-and electrical field-stimulated rat trachea and determine the role of the tracheal epithelium as the possible source of NO involved in these effects. Methods: Isolated carbachol- or electric field-stimulated pre-contracted in vitro male Sprague Dawley rat tracheas (with epithelium intact or denuded) were relaxed with incremental or discrete concentrations of Sal in the presence and absence of the NO synthesis inhibitor L-NAME. Results: Epithelium-denuded tracheas showed a reduced relaxation response to Sal. LNAME (1 mM) similarly decreased the sensitivity of the rat tracheas to Sal in both epithelium-intact and-denuded conditions. In the presence of L-NAME, high concentrations of Sal induced an unexpectedly large relaxation response in carbachol-stimulated rat tracheas with both intact and denuded epithelium. Sal relaxation was also affected by LNAME in electrical field-stimulated epithelium-intact and
\end{abstract}

-denuded tracheas. Conclusion: The results suggest that NO derived from sources other than the epithelium is an important mediator of the Sal-induced relaxation in rat tracheas.

Copyright $\odot 2010$ S. Karger AG, Basel

\section{Introduction}

Nitric oxide (NO) has been well established as a regulator of a multiplicity of physiologic processes [1]. It is involved in platelet aggregation, host defense, neurotransmission, and vascular, gastrointestinal and airway smooth muscle regulation. The physiological role of NO in airway smooth muscle tone is also well documented [2]. NO induces bronchodilation by stimulating processes that lead to the relaxation of the airway smooth muscle cells [1]. It is synthesized from L-arginine by the enzyme NO synthase that exists in different isoforms. Two of these isoforms, endothelial-derived nitric oxide synthase (eNOS) and neuronal nitric oxide synthase (nNOS) occur normally in the airways where they are part of the regulation and availability of NO. Several respiratory functions are known to be regulated by $\mathrm{NO}$ including control of breathing, ventilation-perfusion matching and pulmonary vascular tone [3].

\section{KARGER}

Fax +41613061234 E-Mail karger@karger.ch www.karger.com
(C) 2010 S. Karger AG, Basel

0025-7931/10/0805-0426\$26.00/0

Accessible online at:

www.karger.com/res
Guido E. Santacana, $\mathrm{PhD}$

Department of Physiology, University of Puerto Rico School of Medicine

PO Box 365067, San Juan, PR 00936 (USA)

Tel. +1 787758 2525, ext. 1609, Fax +1 7877530120

E-Mail guido.santacana1@upr.edu 
The existence of NO synthases in the airways [4] plus the multiple regulatory functions that are known to be regulated by NO [3] support its important role in the regulation of airway smooth muscle tone and its possible involvement in the pathophysiology of bronchial asthma. There is now abundant evidence $[3,5,6]$ showing that the bronchodilator effects of NO in health and disease are mediated through the formation of s-nitrosothiols (SNOs). NOS activity leads to the formation of NO which can take the route of SNO formation, principally S-nitrosoglutathione (SNOG) that eventually activates guanylyl cyclase inducing relaxation [5].

Paradoxically from the standpoint of pathophysiology, an increase in exhaled NO has been found to correlate with eosinophylic mucosal infiltration and the severity of asthma [7]. In the general population, atopy, rhinitis and uncontrolled asthma have been positively associated with exhaled NO [8]. This apparent paradox has been explained by Que et al. [9] who found that in asthmatic airways S-nitrosoglutathione reductase (SNOGR) activity is increased, resulting in high $\mathrm{NO}$ levels derived from the breakdown of SNO by SNOGR.

Epithelial-derived NO has been proposed as having a major role in airway smooth muscle relaxation. Thus, the functional integrity of the airway epithelium has been described as a primary factor controlling the physiological responsiveness of airway smooth muscle [10]. Mechanical removal of the epithelium has been shown to increase the sensitivity of isolated tracheal strips to isoprenaline, sodium nitroprusside and adenosine [11]. Nonetheless, inhibition of NO synthesis by N-nitro-L-arginine methyl ester (L-NAME) has shown some contradictory results in which some studies show that L-NAME potentiates agonist induced contractions while other studies report no effects $[12,13]$.

The absence of epithelial-derived $\mathrm{NO}$ as a consequence of epithelial damage has been related to the resulting hypersensitivity of the airways to contractile agents [14]. The fact that there are both hyper-reactive and inflammatory responses in airway smooth muscle during asthma explains the modern treatment alternatives that rely on the use of a combination of relaxing and anti-inflammatory agents. The most widely used airway smooth muscle relaxants in the treatment of asthma are $\beta_{2}$ adrenoceptor agonists like salbutamol (Sal) [15]. Drugs like Sal are thought to act primarily through $\beta_{2}$ adrenergic receptors present in the plasma membrane of airway smooth muscle cells. The binding of Sal to the $\beta_{2}$ receptor induces activation of adenylate cyclase and consequently cAMP generation that eventually leads to a reduction of cytosolic calcium availability resulting in smooth muscle relaxation [16]. A second mechanism of action for these $\beta_{2}$ agonist drugs involving the stimulation of prejunctional receptors of the human [17] and guinea pig trachea nerves [18] has been described. This prejunctional stimulation causes inhibition of the cholinergic-mediated airway smooth muscle contraction and may be an important mechanism by which $\beta_{2}$ agonists induce airway smooth muscle relaxation. A possible third mechanism of action has been described for the relaxant actions of $\beta_{2}$ adrenoceptors in the airways. The mechanism proposes an interaction between NO availability and the action of $\mathrm{B}_{2}$ agonists mediated via the non-adrenergic non-cholinergic neural pathways [18].

It is evident that the role of $\mathrm{NO}$ in the actions of $\beta_{2}$ adrenoceptor agonists in airway smooth muscle is not well understood at the present time. The objective of this study was to investigate how NO synthesis inhibitors modulate the relaxation and contraction of the isolated intact and epithelium-denuded 'in vitro' rat trachea in the presence of the $\beta_{2}$ agonist Sal. We propose that Sal's action on airway smooth muscle may be modulated by endogenous NO-derived sources such as submucosal NCNS nerves, prejunctional nerves, airway epithelium or smooth muscle.

\section{Materials and Methods}

\section{Isolation of the Rat Trachea}

All the animals were used in accordance with the Institutional Animal Care and Use Committee guidelines (IRB approval 3280107).

Male Sprague Dawley rats (250-300 g) were anesthetized with an intraperitoneal lethal dose of pentobarbital sodium (150 mg/ $\mathrm{kg}$ ). As soon as the animal fell into a deep anesthesia, an intact segment $(8-10 \mathrm{~mm})$ of the cervical trachea was surgically removed and cleaned of surrounding tissue. The tracheal segment was conserved intact and placed without further manipulation in a pre-oxygenated Krebs-Ringer bicarbonate solution with the following composition (mM): $\mathrm{NaCl} 117.7, \mathrm{KCl} 4.7, \mathrm{CaCl}_{2} 3.3, \mathrm{Mg}_{2} \mathrm{SO}_{4}$ 2.4, $\mathrm{NaHCO}_{3} 24.8, \mathrm{KH}_{2} \mathrm{PO}_{4} 1.2$ and glucose $10.6(\mathrm{pH}=7.4)$ and bubbled with a gas mixture of $95 \% \mathrm{O}_{2}$ and $5 \% \mathrm{CO}_{2}[19]$.

\section{Isometric Tension Studies}

To determine changes in muscle tension, two stainless steel tissue supports from Rattus suspended the intact tracheal segment in a $25 \mathrm{ml}$ water jacketed organ bath, supplied by Radnoti Glass (Monrovia, Calif., USA). Using SP205 Polyvilene surgical thread (Look SSC, Reading, Pa., USA), the hook of one tissue supporter was secured to the tissue holder and the other hook was connected to a Grass FT.03 force displacement transducer attached to a DC preamplifier model 7P1F (Grass Instruments, West Warwick, R.I., USA). The preamplifier signal was fed into a 
personal computer (Gateway 2000, North South City, S. Dak., USA) containing a PC-LPM-16/PnP data acquisition card (National Instruments, Austin, Tex., USA), and the changes in isometric tension were recorded. The intact trachea was set at an optimal length by equilibrating against a passive load of $2.0 \mathrm{~g}$ at $37^{\circ} \mathrm{C}$ for $30 \mathrm{~min}$.

\section{Preparation of Epithelium-Denuded Tracheas}

To remove the tracheal epithelium a cotton tipped piece of surgical thread was gently scrubbed against the interior of the trachea. This method has been previously shown to remove all traces of the tracheal epithelial layer [20-23]. Histological HE stained slides of epithelium intact and denuded tracheas sections were prepared to ascertain the removal of the epithelium by conventional light microscopy.

\section{Pharmacological Stimulation}

Effect of Sal on the Carbachol-Induced Contraction

For these experiments increasing concentrations of the contractile agonist carbachol (CCh; 0.2 to $10 \mu \mathrm{M}$ ) were added to establish the minimum dose at which a maximal contractile response to CCh was obtained. The maximal contraction was achieved at a concentration of $10^{-7} \mathrm{M}$ CCh. This concentration was used in subsequent experiments to elicit tracheal contraction. Upon achieving the maximal contractile response, the $\beta_{2}$ agonist Sal $\left(10^{-9}\right.$ to $\left.10^{-5} \mathrm{M}\right)$ was added in increasing concentrations and a cumulative dose-response curve was generated. The response to each dose of Sal was expressed as a percentage of the maximal CCh-induced tension. This experiment was done in both intact and epithelium-denuded tracheas.

Effect of L-NAME on the CCh-Induced Contraction and

SAL-Induced Relaxation

To evaluate the contribution of the airway smooth muscle endogenous NO to tracheal contractility, the isolated trachea was first preincubated for 15 min with $1 \mathrm{mM}$ of the NO synthetase inhibitor L-NAME. A dose of $10^{-7} \mathrm{M}$ CCh was added to the incubation medium and the maximal contraction of the intact tracheal segment was determined.

To measure the effect of L-NAME on the Sal-induced relaxation the trachea was first preincubated for 15 min with the NO synthetase inhibitor. This was followed by the addition of $10^{-7} \mathrm{M}$ $\mathrm{CCh}$. After reaching maximal contraction the trachea was then relaxed with increasing concentrations of SAL.

Both of the procedures described above were done in intact and epithelium-denuded tracheas.

\section{Electrical Field Stimulation Studies}

For the electrical field stimulation (EFS) studies the trachea was localized in the organ bath between two equidistant platinum electrodes connected to a Grass S88 square wave DC stimulator. Transmural stimulation using EFS was applied under two conditions: by application of increments in voltage under a constant stimulation frequency of $50 \mathrm{~Hz}$ or application of frequency increments under a constant voltage of $40 \mathrm{VDC}$. In experiments where pre-contraction of the trachea was necessary, increments in voltage or frequency were applied until a constant tension was reached. After the tension remained constant for $1 \mathrm{~min}$ the experimental drug was added in incremental doses to the incubation medium and the response was recorded.

\section{Statistical Analysis}

Dose-response curves for Sal relaxation responses were analyzed using the non-linear regression analysis for sigmoidal doseresponse curves available in the Graph-Pad Prizm program. Data from the electrical field stimulation experiments are presented as mean \pm standard error of the mean. Statistical evaluation of the data was performed using Student's t test. Statistical significance was established at a value of $\mathrm{p}<0.05$.

\section{Results}

Effect of Sal on the CCh Pre-Contracted Intact and Epithelium-Denuded Rat Trachea in the Presence and Absence of L-NAME

The control curves in figures 1 and 2 show the relaxation of the CCh pre-contracted trachea elicited by incremental doses of Sal. In the presence of the epithelium (fig. 1) the maximal Sal-induced relaxation is $35 \%$ of the maximal CCh-induced contraction. The epithelium-denuded trachea (fig. 2) shows a maximal Sal-induced relaxation of $29 \%$. Removal of the epithelium resulted in a reduction of the maximal Sal-induced relaxation of $6 \%$. The $\mathrm{EC}_{50} \mathrm{~s}(-3.998$ vs. -4.966$)$ of the Sal-induced relaxation curves in the intact versus the epithelium-denuded tracheas show that the absence of the epithelium increases the sensitivity of the rat trachea to Sal.

Incubation of the intact trachea with L-NAME resulted in a diminished sensitivity to Sal-induced relaxation with an $\mathrm{EC}_{50}$ of -2.745 in the presence of L-NAME versus -3.998 in its absence (fig. 1). The relaxation response was greatly enhanced at Sal doses higher than $1 \mathrm{mM}$. The effects observed with epithelium removal were similar to those of epithelium intact tracheas with $\mathrm{EC}_{50} \mathrm{~s}$ of -2.794 with L-NAME and -4.966 in its absence (fig. 2). The $\mathrm{EC}_{50} \mathrm{~s}$ of the Sal-induced relaxation curves in the intact and epithelium-denuded tracheas under the influence of LNAME show almost equal values as well as the maximal relaxation effect produced by Sal. A small but significant increase in the maximal baseline tension induced by $\mathrm{CCh}$ was also observed in the presence of L-NAME.

\section{Effects of Sal on the EFS Pre-Contracted Trachea in \\ the Presence and Absence of L-NAME}

Figure 3 shows the result of experiments where the intact rat trachea received transmural stimulation at a frequency of $2 \mathrm{~Hz}$ and a voltage of $40 \mathrm{VDC}$. Under these conditions the maximal contractile response attained was $1.807 \pm 0.17 \mathrm{~g}$. After the addition of Sal $(1 \mu \mathrm{M})$ the tension was $1.338 \pm 0.27 \mathrm{~g}$ indicating a $26 \%$ reduction in the tracheal response to EFS. 


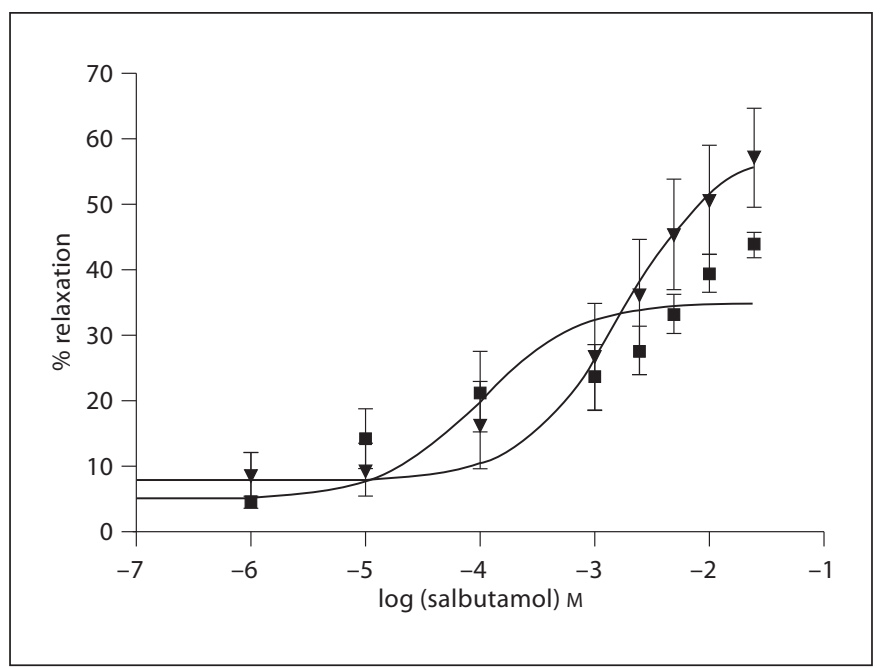

Fig. 1. SA induced relaxation in CCh pre-contracted epithelium intact trachea. $\boldsymbol{\square}=$ Control; $\boldsymbol{\nabla}=$ L-NAME. $\mathrm{EC}_{50}($ control $)=$ -3.998; $\mathrm{EC}_{50}(\mathrm{~L}-\mathrm{NAME})=-2.745$.

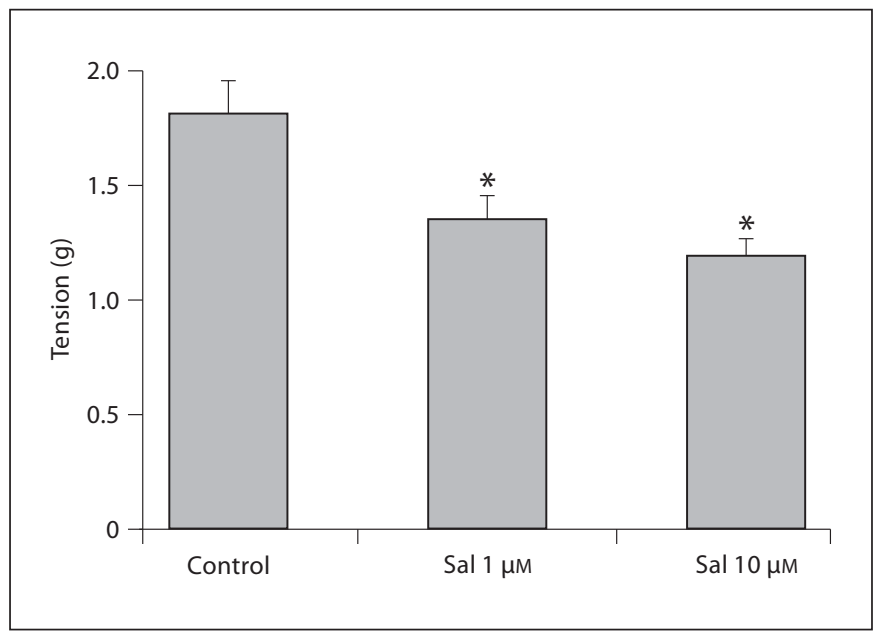

Fig. 3. EFS stimulation of the intact trachea in the absence and presence of SA $(1$ and $10 \mu \mathrm{M}){ }^{*} \mathrm{p} \leq 0.05, \mathrm{n}=6$.

When the concentration of Sal was increased to $10 \mu \mathrm{M}$, the tension induced by EFS was reduced further to a value of $1.182 \pm 0.18 \mathrm{~g}$, that is $35 \%$ less tension than the control value and $12 \%$ less tension than with $1 \mu \mathrm{M} \mathrm{Sal}$ (fig. 4), indicating that the relaxation response to Sal of the EFS pre-contracted intact rat trachea is concentration dependent.

Figure 4 shows that in the presence of $1 \mathrm{mM} \mathrm{L-NAME}$ the EFS-induced contraction of the rat trachea was reduced from $1.74 \pm 0.14$ to $1.50 \pm 0.14 \mathrm{~g}$. This is a $14 \%$

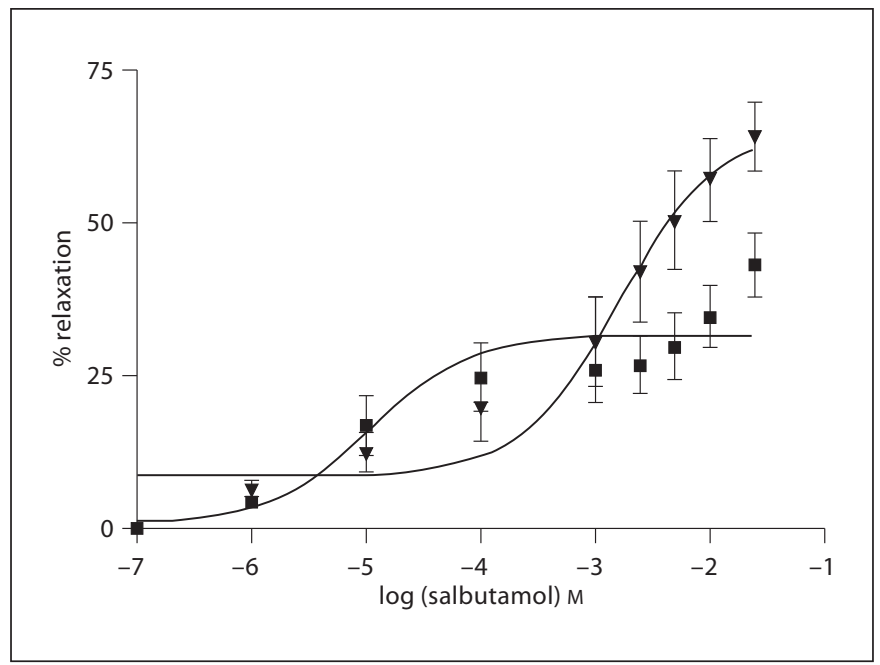

Fig. 2. SA induced relaxation in CCh pre-contracted epitheliumdenuded trachea. $\mathbf{\square}=$ Control; $\boldsymbol{\nabla}=$ L-NAME. EC $_{50}$ (control) $=$ $-4.966 ; \mathrm{EC}_{50}(\mathrm{~L}-\mathrm{NAME})=-2.794$.

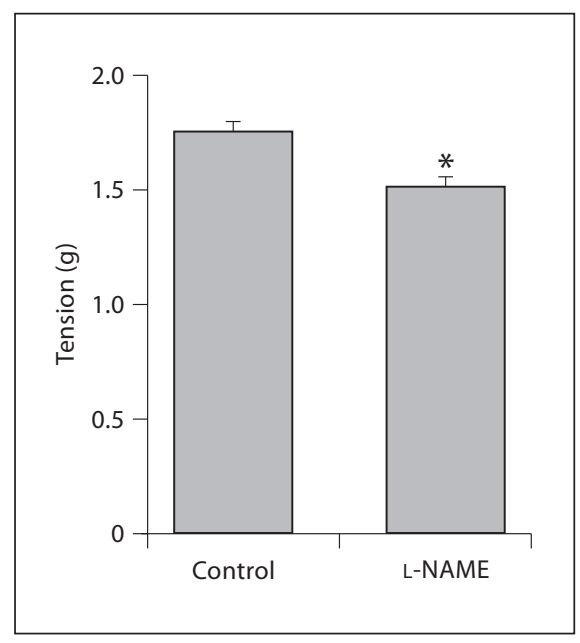

Fig. 4. EFS induced contraction of the rat trachea in the presence and absence of $1 \mathrm{mM} \mathrm{L-NAME}$. ${ }^{*} \mathrm{p} \leq 0.05, \mathrm{n}=6$.

decrease in tension as a result of the inhibition of NO synthesis by L-NAME. Although this effect was not significant, it was unexpected.

Pre-incubation of the rat trachea with L-NAME $(1 \mathrm{mM})$ reduced to non-significant levels the relaxation effect of Sal (1 or $10 \mu \mathrm{M})$ on the EFS-induced contraction which is in accordance to the effect expected after inhibition of NO synthesis and indicates that NO synthesis is a requisite for the Sal-induced relaxation of rat trachea (fig. 5). 


\section{Discussion}

The isolated 'in vitro' rat tracheal muscle produces a contractile response to $\mathrm{CCh}\left(10^{-7} \mathrm{M}\right)$ that reaches a plateau or peak value in $1 \mathrm{~min}$. This contraction can be sustained for a period of time longer than $60 \mathrm{~min}$ before any significant change in the level of tension can be observed. Sal, added in incremental doses after the maximal CCh contraction was attained, produced a relaxation response that reached its maximum per dose in a period of $5 \mathrm{~min}$. This Sal-induced relaxation is observed in guinea pig isolated trachealis [11] and has been proposed to be primarily elicited by activation of the $\beta_{2}$ adrenergic receptors of the tracheal smooth muscle followed by an increase in the synthesis of CAMP with consequent cytosolic calcium sequestration inside the smooth muscle cell leading to less availability of calcium for the contractile apparatus of the cell and relaxation $[16,18]$.

In this study we show that Sal relaxed the CCh precontracted trachea in a concentration dependent manner with the maximal effect elicited at a Sal concentration of $1 \mathrm{~mm}$ (fig. 1). Removal of the epithelial layer produced a small but significant increase in sensitivity of the tracheal response to Sal as indicated by the $\mathrm{EC}_{50} \mathrm{~s}$ of $9.9 \times 10^{-5}$ and $4.4 \times 10^{-5} \mathrm{M}$ for the control and epithelium-denuded tracheas, respectively (fig. 1,2 ). This effect could be ascribed to the absence of the barrier represented by the epithelium, thus making the drug more accessible to the deeper muscle layers in the tissue but epithelium relaxing factors like NO could be also implicated in this increased sensitivity as reported by several authors [19-25]. The epithelial layer has been previously shown to have very little if any effect on the $\beta_{2}$ agonist-induced relaxation of the guinea pig trachea under similar experimental conditions [26]. Addition of the NO synthesis inhibitor LNAME to the incubation medium for $1 \mathrm{~h}$ resulted in a significant reduction in the sensitivity to Sal-induced relaxation of the CCh pre-contracted trachea (fig. 1). A similar reduction in sensitivity to Sal was also observed in the response of the epithelium-denuded trachea to Sal in the presence of L-NAME (fig. 2). These results strongly suggest that the relaxation induced by Sal in the CCh precontracted trachea is in part mediated by the synthesis and release of $\mathrm{NO}$ indicating the possible influence of Sal in the cGMP pathway that mediate the effects of $\mathrm{NO}$ in the regulation of airway smooth muscle tone. The experiments also suggest that the source of the NO is not the epithelial layer since removal of the epithelium had no effect on the Sal-induced relaxation of the L-NAME treated trachea. Paradoxical effects in which Sal, at concentra-

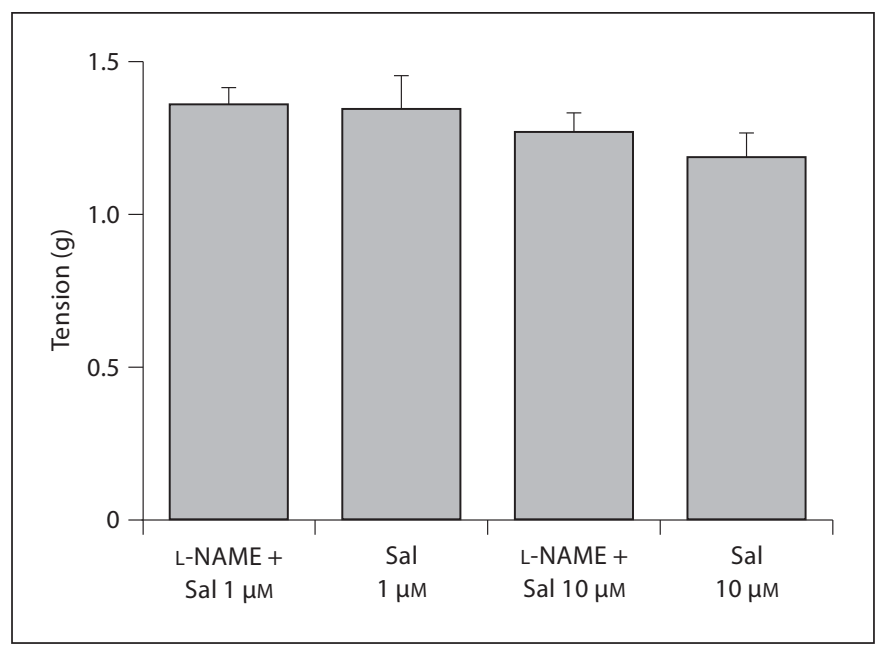

Fig. 5. EFS induced contraction of the rat trachea in the presence of SA $(1$ and $10 \mu \mathrm{M})$ after incubation in $1 \mathrm{mM} \mathrm{L-NAME}$.

tions higher than $10^{-5} \mathrm{M}$, exacerbates the acetylcholine induced contraction of human and guinea pig airways have been reported and related to effects on the drug on calcium channels [19]. We did not observe these effects at high concentrations in our control experiments but under the influence of L-NAME there was a definite potentiation of the Sal-induced relaxation in both intact and epithelium-denuded tracheas.

The tracheal segments where the pre-contraction was induced by transmural (EFS) stimulation also showed a dose dependent response to Sal $(1$ and $10 \mu \mathrm{M})$ induced relaxation as observed in figure 3 . Transmural stimulation in the rat trachea is primordially elicited by the endogenous release of the cholinergic neurotransmitter acetylcholine and can be inhibited by the cholinergic muscarinic receptor antagonist atropine [20]. Incubation of the rat trachea with L-NAME (1 mM) followed by transmural stimulation in the presence of the NO synthesis inhibitor resulted in a small but significant reduction in the maximal response of the rat trachea to EFS (fig. 4). This was an unexpected result that may be related to a prejunctional neural effect of L-NAME. One possible explanation is the relation of NOS to the production of superoxides capable of inducing contractile responses in smooth muscle $[27,28]$. Inhibition of NOS could result in the reduction of superoxide generation and the diminished contractile response to EFS observed in the LNAME-incubated rat trachea. There is also the possibility of inhibition of serotonin release from prejunctional nerves by L-NAME, an effect that has been described by 
Garcia et al. [29] for sympathetic outflow studies in pithed rats. Nonetheless, when the rat trachea was pre-incubated in L-NAME and contracted with EFS, the addition of 1 or $10 \mu \mathrm{M}$ Sal could not elicit a significant relaxation response (fig. 5). This indicates that indeed the presence of the NO synthesis inhibitor is blocking the relaxation effects of Sal under EFS stimulation, implicating NO in the Sal induced relaxation under conditions where endogenous activation of the contractile response of rat trachea is elicited (fig. 5). It also indicates that the NOS inhibiting action of L-NAME is taking place as expected.

Our data correlates with the results obtained by Wang et al. [30] using isolated rat aortic rings in which NO was shown to be involved in the actions of Sal. The fact that L-NAME significantly reduced the sensitivity of the rat trachea to Sal in both intact and epithelium-denuded tracheas shows that there is a definite involvement of NO in the actions of Sal in airway smooth muscle. This is further supported by the inability of SA to relax EFS precontracted tracheas in the presence of L-NAME. The results tend to support non-epithelial sources as main factors in the NO-dependent actions of Sal. Further studies are needed to ascertain the level of involvement of NO in the relaxant actions of the $\beta_{2}$ agonist Sal in the airways. It is also important to recognize the fact that this study has focused mainly in the larger airways. $\beta$ agonists show either variable or poor results as small airway smooth muscle relaxants $[31,32]$. Nonetheless, studies have demonstrated that the significant number of peripheral small airways contribute much less than the large airways to the total resistance to airflow in the tracheobronchial tree [33-35] making large airways an important target of $\beta$ agonists.

\section{References}

1 Ricciardolo FLM, Sterk PJ, Gaston B, Folkerts G: Nitric oxide in health and disease of the respiratory system. Physiol Rev 2004:84: 731-765.

2 Hamad AM, Clayton A, Islam B, Knox AJ: Guanylyl cyclases, nitric oxide, natriuretic peptides, and airway smooth muscle function. Am J Physiol Lung Cell Mol Physiol 2003:285:L973-L983.

- 3 Gaston B, Reilly J, Drazen JM, Fackler J, Ramdev P, Arnelle D, Mullins ME, Sugarbaker DJ, Chee C, Singel DJ, Loscalzo J, Stamler JS: Endogenous nitrogen oxides and bronchodilator S-nitrosothiols in human airways. Proc Natl Acad Sci 1993;90:1095710961

-4 Meurs H, Hamer MAM, Pethe S, Vadon-Le Goff S, Boucher JL, Zaagsma J: Modulation of cholinergic airway reactivity and nitric oxide production by endogenous arginase activity. Br J Pharmacol 2000:130:17931798.

5 Mayer B, Pfeiffer S, Schrammel A, Koesling D, Schmidt K, Brunner F: A new pathway of nitric oxide/cyclic gmp signaling involving s-nitrosoglutathione. J Biol Chem 1998;273: 3264-3270.

-6 Fang K, Johns R, MacDonald T, Kinter M, Gaston B: S-nitrosoglutathione breakdown prevents airway smooth muscle relaxation in the guinea pig. Am J Physiol 2000;279:L716L721.

7 Payne DN, Adock IM, Wilson NM, Oates T, Scallan M, Bush A: Relationship between exhaled nitric oxide and mucosal eosinophilic inflammation in children with difficult asthma, after treatment with oral prednisolone. Am J Crit Care Med 2001;164:1376-1381.
8 Bommarito L, Migliore E, Bugiani M, Heffler E, Guida G, Bucca C, de Marco R, Rolla G: Exhaled nitric oxide in a population sample of adults. Respiration 2008;75:386-392.

\9 Que LG, Yang Z, Stamler JS, Lugogo Njira, Kraft M: S-nitrosoglutathione reductase, an important regulator in human asthma. Am J Respir Crit Care Med 2009;180:226-231.

10 Morrison KJ, Gao Y, Vanhoutte PM: Epithelial modulation of airway smooth muscle. Am J Physiol 1990 258:L254-L252.

11 Farmer SG, Fedan JS, Hay DWP, Raeburn D: The effects of epithelium removal on the sensitivity of guinea-pig isolated trachealis to bronchodilator drugs. Br J Pharmac 1986:89: 407-414.

12 Xiang Y: Endothelium-derived nitric oxide partially mediates salbutamol-induced vasodilations. Brit J Pharmacol Therapeutics 1993;250:335-340.

13 Aizawa H, Tanaka H, Sakai J, Takata S, Hara $\mathrm{N}$, Ito Y: L-Name sensitive and insensitive nonadrenergic noncholinergic relaxation of cat airway in vivo and in vitro. Eur Res J 1997; 10:314-321.

14 Barnes PJ: Nitric oxide and asthmatic inflammation. Immunol 1995;16:128-130.

15 Proskocil BJ, Fryer AD: Beta ${ }_{2}$-agonist and anticholinergic drugs in the treatment of lung disease. Proc Am Thorac Soc 2005: 2:305-310

16 Lefkowitz RJ, Caron M, Michel T, Stadel J: Mechanims of hormone receptor-effector coupling: the B adrenergic receptor and adenylate cyclase. Fed Proc 1982;41:26642670 .
17 Rhoden KJ: Inhibition of cholinergic neurotransmission in human airways by $\beta_{2}$-adrenoceptors. J Appl Physiol 1988;65:700-705.

18 Wessler I, Reinheimer T, Brunn G, Anderson GP, Maclagan J, Racké K: Beta-adrenoceptors mediate inhibition of $[3 \mathrm{H}]$-acetylcholine release from the isolated rat and guinea-pig trachea: role of the airway mucosa and prostaglandins. Br J Pharmacol 1994:113:12211230 .

19 Girodet PO, Berger P, Martinez B, Marthan R, Advenier C, Molimard M: Paradoxal effect of salbutamol in an in vitro model of bronchoprotection. Fundam Clin Pharmacol 2005:19:179-186.

20 Gonzalez O, Santacana G: Effect of airway epithelium on the contraction of the rat isolated trachea under conditions of low temperature. Respiration 2000;67:439-442.

21 Flavahan NA, Aarhus LL, Rimele TJ, Vanhoutte PM: Respiratory epithelium inhibits bronchial smooth muscle tone. J Appl Physiol 1985;58:834-838.

22 Hay DW, Farmer SG, Raeburn D, Robinson VA, Fleming WW, Fedan JS: Airway epithelium modulates the reactivity of guinea-pig respiratory smooth muscle. Eur J Pharmacol 1986;129:11-18.

23 Barnes PJ, Cuss FM, Palmer JB: The effect of airway epithelium on smooth muscle contractility in bovine trachea. Br J Pharmacol 1985;86:685-691.

24 Raeburn D, Hay DW, Farmer SG, Fedan JS: Epithelium removal increases the reactivity of human isolated tracheal muscle to methacholine and reduces the effect of verapamil. Eur J Pharmacol 1986;123:451-453. 
-25 Nijkamp FP, van der Linde HJ, Folkerts G: Nitric oxide synthesis inhibitors induce airway hyperresponsiveness in the guinea pig in vivo and in vitro: role of the epithelium. Am Rev Respir Dis 1993;148:727-734.

-26 Daffonchio L, Hernandez A, Martinotti E, Omici C: B-adrenoceptor reactivity after epithelium removal in guinea pig trachea in vitro. Pharmacol Res 1990;22:49-58.

-27 Chen C-A, Druhan LJ, Varadharaj S, Chen YR, Zweier JL: Phosphorilation of endothelial nitric oxide synthase regulates superoxide generation from the enzyme. J Biol Chem 2008;283:27038-27047.
28 de Boer J, Meurs H, Flendrig L, Koopal M, Zaagsma J: Role of nitric oxide and superoxide in allergen-induced airway hyperreactivity after the late asthmatic reaction in guinea pigs. Br J Pharmacol 2001;133:1235-1242.

29 Garcia M, Moran A, Luisa Martin M, Barthelemebs M, San Roman L: The nitric oxide synthesis/pathway mediates the inhibitory serotoninergic responses of the pressor effect elicited by sympathetic stimulation in diabetic pithed rats. Eur J Pharmacol 2006;537: 126-134.

30 Wang YX, Poon KS, Randall DJ, Pang: Endothelium-derived nitric oxide partially mediates salbutamol-induced vasodilatations. CC Eur J Pharmacol 1993;250:335-340.

-31 Finney MJB: Effects of bronchoconstrictors and bronchodilators on a novel human small airway preparation. Br J Pharmacol 1985;85; 29-36.
2 Skogvall S, Berglund M, Dalence-Guzman MF, Svensson K, Jonsson P, Persson CG, Sterner O: Effects of capsazepine on human small airway responsiveness unravel a novel class of bronchorelaxants. Pulm Pharmacol Ther 2007;20;273-280.

33 Hogg JC: Pathophysiology of airflow limitation in chronic obstructive disease. Lancet 2004;364:985-996.

>34 King GG, Downie SR, Vernbanck S, Thorpe CW, Berend N, Salome CM, Thompson B: Effects of methacholine on small airway function measured by forced oscillation technique and multiple breath nitrogen washout in normal subjects. Respir Physiol Neurobiol 2005;148:165-177.

35 Roche WR: Inflammatory and structural changes in the small airways in bronchial asthma. Am J Respir Crit Care Med 1998; 157:S191-S194. 